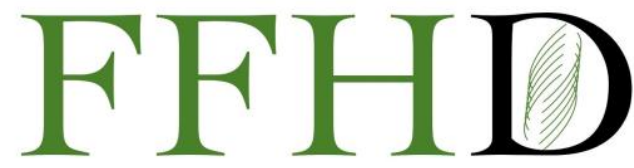

Functional Foods in Health and Disease

\title{
Effect of 12 weeks of continuous burdock sprout extract intake on oxidized LDL: A placebo-controlled, randomized, double-blind, parallel-groups study
}

\author{
Naohito Ito $^{1}$, Akiko Ohno ${ }^{2}$, Satoshi Yomoda ${ }^{2}$, Satoshi Inamasu' ${ }^{2}$ Hiroki Hattori ${ }^{1}$ and Jun Nishihira ${ }^{1 *}$ \\ ${ }^{1}$ Center for Health Information Sciences, Hokkaido Information University Nishinopporo 59-2, Ebetsu City, Hokkaido; ${ }^{2}$ R\&D \\ Innovation Group, Corporate Planning Division, Kracie Holdings, Ltd.
}

*Corresponding author: Jun Nishihira, MD, PhD, Department of Medical Management and Informatics, Hokkaido Information University, Nishi Nopporo 59-2, Ebetsu 069-8585 Hokkaido, Japan

Submission Date: July $2^{\text {nd }}, 2021$; Acceptance Date: August 19 ${ }^{\text {th }}, 2021$; Publication Date: September $8^{\text {th }}, 2021$

Please cite this as: Ito N., Ohno A., Yomoda S., Inamasu S., Hattori H., Nishihira J. Effect of 12 weeks of continuous burdock sprout extract intake on oxidized LDL: A placebo-controlled, randomized, double-blind, parallel-groups study. Functional Foods in Health and Disease 2021. 11(9): 456-472. DOI: https://www.doi.org/10.31989/ffhd.v11i9.820

\begin{abstract}
Background: Arctigenin, a lignan polyphenol found in burdock sprout, is a potent activator of AMP-activated protein kinase (AMPK) and has beneficial effects on metabolic disorders in mice. This highlights the potential value of arctigenin for the reduction of oxidized LDL (oxLDL) by activation of AMPK in human, but not well elucidated.

Methods: We conducted a placebo-controlled, randomized, double-blind, parallel-groups study in healthy adults to investigate the effects of 12 weeks of a continuous intake of burdock sprout extract enriched with arctigenin on lipid metabolism. The subjects were 100 Japanese individuals with low-density lipoprotein cholesterol (LDL-C) < $140 \mathrm{mg} / \mathrm{dl}$ and a body mass index (BMI) of $23-30 \mathrm{~kg} / \mathrm{m}^{2}$ who were randomly assigned to receive either burdock sprout extract (test food) or a placebo. The test food was encapsulated burdock sprout extract containing approximately 40 mg of arctigenin equivalent per day, and the placebo capsules contained no burdock sprout extract. The subjects consumed the assigned foods daily for 12 weeks.
\end{abstract}




\section{Mechanism of hepatic fibrosis by oxidized LDL and mechanism of action of arctigenin (hypothesis)}

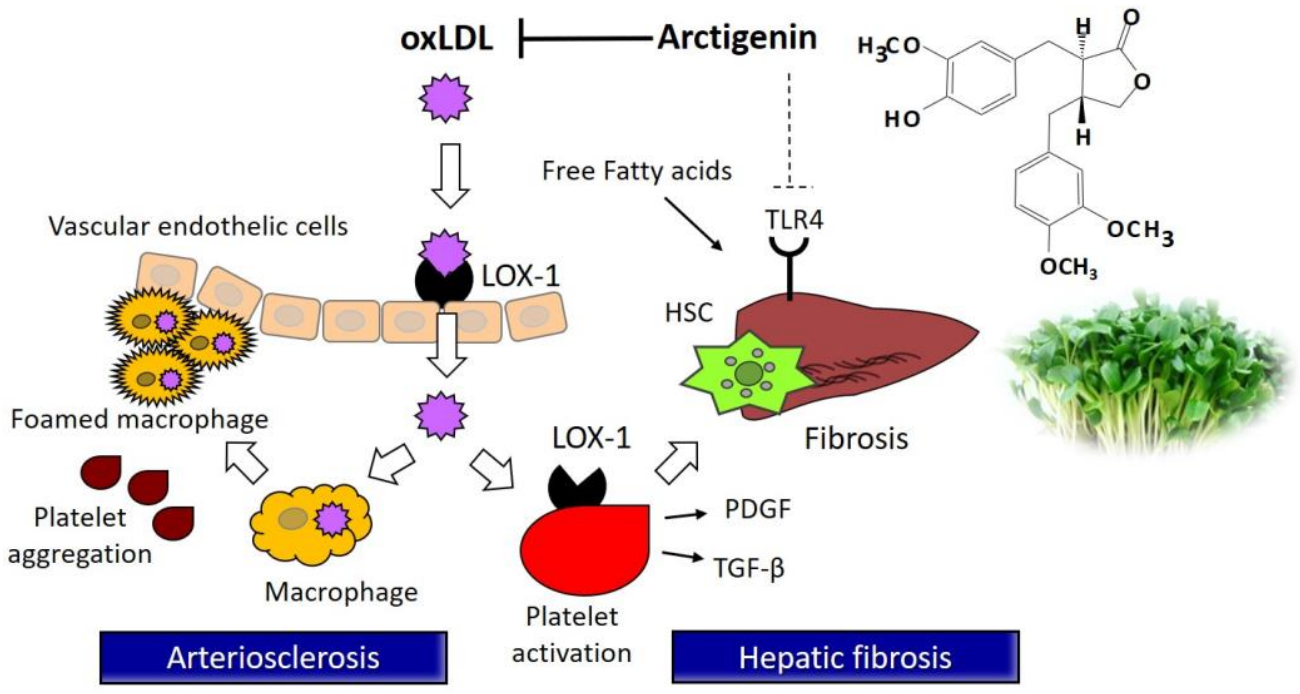

Results: The test food significantly suppressed oxLDL after 12 weeks in subjects with a body mass index (BMI) of $25 \mathrm{~kg} / \mathrm{m}^{2}$ or higher. The test food inhibited an increase in the FIB-4 index (liver fibrosis index), an indicator of hepatic fibrosis. Pearson's correlation coefficient analysis revealed a significant correlation between oxLDL and FIB-4 index changes, suggesting that the test food suppressed the rise in the FIB-4 index by controlling oxLDL while maintaining the platelet count.

Conclusions: We conclude that the consumption of a burdock sprout supplement containing arctigenin is effective in suppressing oxLDL, which may prevent liver fibrosis.

\section{Clinical trial registration: UMIN000036664}

Keywords: arctigenin, burdock sprout, oxidized LDL, FIB-4 index, randomized controlled study

(CFFC 2021. This is an Open Access article distributed under the terms of the Creative Commons Attribution 4.0 License (http://creativecommons.org/licenses/by/4.0)

\section{INTRODUCTION}

Dyslipidemia is a major risk factor for the development of coronary heart and cerebrovascular diseases. The number of patients with this condition is increasing, according to the Patient Survey of the Ministry of Health, Labor and Welfare in 2014. The number of patients undergoing treatment has increased to 211 million. A review of dietary habits is essential for preventing dyslipidemia, and there is a growing demand for the use of functional foods to mitigate against disordered lipid metabolism.

Various factors such as metabolic dysfunction, inflammation, and oxidative stress are intricately linked in the progression of arteriosclerosis, in which the oxidation of LDL-C (low-density lipoprotein cholesterol) plays an important role. $\mathrm{LDL}-\mathrm{C}$ undergoes oxidative reactions in the vascular walls and is readily taken up by macrophages. 
Macrophages transform into foam cells and promote plaque progression and consequently, vascular endothelial dysfunction due to the migration of vascular smooth muscle cells. The production of inflammatory cytokines released by migrating immune cells aggravates systemic vascular-related disorders. Therefore, LDL-C is recognized as being associated with deteriorating cholesterol and the promotion of arteriosclerosis.

The concept that the oxidation of lipoproteins is central in the pathogenesis of atherosclerosis was first reported by in vitro studies and, subsequently, through experimental models of atherosclerosis. In recent years, advances in analytical methods, particularly using monoclonal antibodies against oxidized LDL (oxLDL), revealed the presence of oxLDL in the circulation, and its role as a biomarker became popular [1]. OxLDL is a generic term for oxidized and denatured LDL particles that directly affect arteriosclerosis. There are various oxidative reaction sites, including apoprotein $B$ and phospholipids, and the degree of oxidation varies. Clinically, malondialdehyde-induced apo-B (MDA-LDL) is often measured as oxLDL, a circulatory oxidative LDL. MDA-LDL was certified as a health-insurance indicator in 2008, and MDA-LDL has been used as a surrogate marker for oxLDL in the circulation. Recently, several studies have revealed that arteriosclerosis is associated with fatty liver and liver fibrosis. Using the pulse-wave velocity (PWV) test, Kokubo et al. demonstrated that arteriosclerosis is significantly correlated with fibrosis prediction in NAFLD (nonalcoholic fatty liver disease) according to the FIB-4 index [2]. Accordingly, studies on the relation between the development and progression of arteriosclerosis caused by oxLDL and liver fibrosis are emerging.

Burdock sprout, the shoot immediately prepared after germination, is rich in polyphenols. The major constituents, arctigenin and its sugar complex arctiin, are lignans that are often detected in burdock (Arctium lappa L.) seeds [3]. They have various biochemical activities, including antioxidative, anti-inflammatory and anticancer activities. Trace amounts of arctigenin are also present in burdock roots and leaves [4-5]. Arctiin is rapidly transformed to arctigenin in the large intestinal flora [6]. Arctigenin has various effects, including antioxidant [7-8], anti-inflammatory [9-10], antiviral [11], and immunomodulatory effects [12]. Moreover, continuous intake of arctigenin has been shown to lower high-fat dietinduced body weight and the serum lipid level [13]. Furthermore, arctigenin was found to alleviate fat deposition in the liver and reduce epididymal fat accumulation. Arctigenin was also shown to suppress lipogenesis and lipolysis and promote fatty acid betaoxidation. Most importantly, arctigenin increases the phosphorylation of AMP-activated protein kinase (AMPK) and regulates its downstream genes acetyl CoA carboxylase (ACC), peroxisome proliferator-activated receptor $\gamma$ (PPAR $\gamma)$, and sterol regulatory element-binding transcription factor 1 (SREBP1c) [13-14]. Burdock sprout is bitter and is difficult to ingest in a large enough quantity through foods for the functions of arctigenin to be exerted, but we can ingest it in the form of burdock sprout extract. We reported that dietary administration of burdock sprout extract (about $10 \%$ arctigenin content) to high-fat, diet-challenged mice resulted in obesitysuppressive and lipid-lowering effects [15].

In this study, we conducted a placebo-controlled, randomized, double-blind, parallel-groups study to examine the effects of burdock sprout extract on lipid metabolism. We set the blood LDL-C and oxLDL (MDA-LDL) contents as the primary and secondary endpoints, respectively. We found that burdock sprout extract suppressed an increase in inhibition of oxLDL in subjects with a body mass index (BMI) of $25 \mathrm{~kg} / \mathrm{m}^{2}$ or higher. For further analysis, we investigated the association of oxLDL with serum lipids, the visceral fat area, the L/S ratio (ratio of liver to spleen) (fatty liver index), and other laboratory markers. Concerning these biomarkers, reliable and 
inexpensive noninvasive markers of hepatic fibrosis are required for patients with nonalcoholic fatty liver disease (NAFLD). To fulfil this need, the FIB-4 index was developed and recommended as a noninvasive panel to stage liver disease in subjects with chronic liver diseases [16-18]. The FIB-4 index is calculated from hematology examinations and assesses associations with oxLDL. In this study, we investigated the degree of hepatic fibrosis using the FIB-4 index and examined the effects of arctigenin on the progress of hepatic fibrosis.

\section{METHODS}

Study Design: This study was conducted as a placebocontrolled, randomized, double-blind, parallel-groups study. This study conformed to the Ethics Guidelines for Medical Research on Humans (February 28, 2017, Ministry of Education, Culture, Sports, Science and Technology, Partial Revision) and the Declaration of Helsinki (October 2013, Revision of World Medical Association Fortaleza General Assembly). The protocol of the clinical trial was approved by the Bioethics Committee of Hokkaido Information University (April 22, 2019, Approval No.: 2019-01) and registered with the University Hospital Medical Information Network (UMIN) before the start of the test (Registration Date: 20195 July, UMIN Test ID: UMIN000036664). This study was conducted from May to October 2019 at the Center of Health Information Science, Hokkaido Information University (Ebetsu City, Hokkaido), with written consent obtained from all subjects prior to participation in the study.

Selection of Subjects: We performed screening for 226 volunteer enrollees managed by the Center of Health Information Science, Hokkaido Information University. Inclusion criteria were men and women aged 30-65 years at the time of informed consent, with LDL-C $<140 \mathrm{mg} / \mathrm{dl}$ at the time of screening, and a BMI of $23-30 \mathrm{~kg} / \mathrm{m}^{2}$. One hundred individuals were approved by the primary investigator as being appropriate. Participants were excluded from the study if they met any of the exclusion criteria, which included mainly (1) those with dyslipidemia or diabetes mellitus or cardiovascular cerebrovascular diseases; (2) those who had undergone major gastrointestinal surgery; (3) those who may have severe allergic reactions to drugs or foods; (4) heavy smokers and alcohol users; and (5) women who were pregnant or possibly pregnant/breastfeeding.

To calculate the desirable sample size, we set the between-group difference in LDL-C changes as $9 \mathrm{mg} / \mathrm{dL}$ based on the test food intake, assuming a standard deviation in LDL-C of $12.5 \mathrm{mg} / \mathrm{dL}$ for each group. In this case, to confirm a two-sided significance level of $5 \%$ and a power of $80 \%$ in an independent two-sample t-test analysis, we needed 43 subjects per group. Accordingly, the subject number for enrollment was 50 in each group, and the total subject number was set as 100.

We randomly allocated subjects into two groups, test foods and placebo, using a stratified replacement block method considering gender, age composition, LDL-C at screening, and BMI as stratification factors created by a third party, the Denshi Gakuen Kyushu Media Education Center (Ebetsu City, Hokkaido). Any staff involved in the examination, such as the chief investigator, nurses, and coordinator, were not allowed to conduct assignment work or be given related information during the study period.

Preparation of Test Food: Kracie Holdings Ltd. kindly provided the test and placebo foods. The test food was a burdock sprout extract capsule containing approximately $40 \mathrm{mg}$ of arctigenin equivalent per day, and the placebo was a burdock sprout extract-free capsule. We prepared the burdock sprout extract through ethanol extraction of dried burdock sprout. Sankyo Co., Ltd. (Shizuoka, Japan) prepared both the test and placebo foods as indistinguishable formulations of approximately $300 \mathrm{mg}$ 
per capsule.

We describe the nutrient content and arctigenin equivalents consumed daily in the test and placebo foods in Table 1 . The analysis was conducted by the Japan Food Analysis Center (Tokyo, Japan). We prepared arctigenin
$\left(\mathrm{C}_{21} \mathrm{H}_{24} \mathrm{O}_{6}\right.$ molecular weight: 372.42$)$ and $\operatorname{arctiin}\left(\mathrm{C}_{27} \mathrm{H}_{34} \mathrm{O}_{11}\right.$ molecular weight: 534.56) by pulverizing the capsular contents with a mortar with $50 \% \mathrm{MeOH}$ for 30 min and then measured solutions using HPLC using methods provided by food providers.

Table 1. Nutrient compositions of the placebo and test foods based on daily consumption.

\begin{tabular}{|c|c|c|}
\hline & Placebo food & Test food \\
\hline Caloric (kcal) & 2.3 & 2.8 \\
\hline Water content (mg) & 36.8 & 50.6 \\
\hline Proteins (mg) & 1.2 & 78.9 \\
\hline Lipid (mg) & 10.6 & 35.7 \\
\hline Carbohydrates (mg) & 562 & 539 \\
\hline Ash content (mg) & 13.7 & 40.2 \\
\hline $\mathrm{Na}(\mathrm{mg})$ & 0.5 & 1.1 \\
\hline Arctigenin equivalent (mg) & - & 39.3 \\
\hline
\end{tabular}

Amount converted to arctigenin equivalent $(\mathrm{mg})=\operatorname{arctigenin}(\mathrm{mg})+\operatorname{arctiin}(\mathrm{mg}) * 372.42 / 534.56$

Study Procedure: Subjects were requested to consume two capsules of the test or placebo food once daily for 12 weeks from the first day of the study. We set the intake time as just before dinner, but intake after food was also allowed when subjects forgot to consume it beforehand. Medical interviews included measurements of vital signs and body composition, blood chemistry, and questionnaires at weeks $0,4,8$, and 12 . We also performed abdominal computed tomography (CT) before the start of supplementation and after 12 weeks. No food and drink other than water were allowed after 9 p.m. on the day before each visit.

During the study, intake of pharmaceutical drugs was essentially prohibited, and consumption of commercially available functional foods and supplements, including foods for specified health purposes, was also not permitted. In daily life, we advised the subjects to avoid irregular activities (such as sleep deprivation, binge eating), maintain a stable everyday lifestyle in terms of diet and exercise prior to participating in the study, and abstain from unbiased food intake patterns, such as consuming large or frequent amounts of specific foods.

Laboratory Tests: We assessed the primary outcome as the LDL-C, and the secondary outcomes as the total cholesterol (TC), triglyceride (TG), HDL-cholesterol (HDL-C), and oxLDL, as well as the body weight, BMI, L/S ratio, visceral fat area, total abdominal fat area, and abdominal subcutaneous fat area. To assess food safety, we measured vital signs including blood pressure (BP) and the pulse rate at each visit. General blood parameters such as white blood cell (WBC), red blood cell (RBC), hemoglobin $(\mathrm{Hb})$, hematocrit $(\mathrm{Ht})$, platelets $(\mathrm{Plt})$, mean corpuscular hemoglobin $(\mathrm{MCH})$, mean corpuscular hemoglobin concentration $(\mathrm{MCHC})$, and mean corpuscular volume (MCV), liver function parameters such as aspartate 
aminotransferase (AST), alanine aminotransferase (ALT), $Y$ glutamic pyruvic transaminase (GTP), alkaline phosphatase (ALP), lactate dehydrogenase (LDH), total bilirubin (T-Bil), and albumin (Alb), kidney function parameters including blood urea nitrogen (BUN), creatinine (CRE), and uric acid (UA), and blood glucose parameters such as fasting glucose and hemoglobin A1c ( $\mathrm{HbA1c})$, and conducted a urinalysis $(\mathrm{pH}$, qualitative glucose, qualitative protein, occult blood, urobilinogen, ketone bodies, bilirubin). Laboratory tests analyzed by Sapporo Clinical Laboratory Center Co., Ltd. Abdominal CTs were performed at the Central $\mathrm{Cl}$ Clinic (Sapporo) to measure the $\mathrm{L} / \mathrm{S}$ ratio, visceral fat area.

Assessment of Safety: We evaluated the incidence of adverse events from the start of the intake of test foods to the end of the entire clinical trial, including physical signs and abnormal change laboratory parameter. We thoroughly linked symptoms to the date of onset, severity, continuation or discontinuation, procedures, causality with the test food, and date of the outcome for each adverse event. The severity of adverse events and their causality with the test food were classified according to protocol criteria set by the investigator.

Statistical Analysis: Statistical analyses were performed using SPSS Statistics 19 (IBM, Armonk, NY, USA). We performed an independent samples t-test for continuous parametric data between the two groups. Fisher test was used for categorical data, and Mann-Whitney test was used for continuous non-parametric data (i.e., intake rate). The data normality was checked using histograms. Changes in subject values were analyzed using repeatedmeasures two-way ANOVA between the groups. In the subgroup analysis, we analyzed subjects with a BMI of 25 $\mathrm{kg} / \mathrm{m}^{2}$ or higher (Overweight (not obese) range) and subjects with a BMI of less than $25 \mathrm{~kg} / \mathrm{m}^{2}$ (healthy weight range). Values are presented in the tables as the means \pm standard deviations. In all analyses, two-tail tests of statistical significance were used and $p$-value of $<0.05$ was considered significant.

In an additional analysis, we examined correlations between the two variables by Pearson's correlation analysis. The FIB-4 index, used in the additional analysis, was calculated from the age, AST and ALT concentrations, and platelet count (PIt) according to the following formula. FIB-4 index $=\left(\right.$ age $[$ year $] \times$ AST $[$ IU/L] $) /\left(\right.$ Plt $\left[10^{9} /\right.$ L $] \times$ ALT $\left.[\mathrm{IU} / \mathrm{L}]^{\wedge} 1 / 2\right)[16]$.

\section{RESULTS}

Subject Characteristics: One hundred individuals participated in this study based on the inclusion and exclusion criteria. A total of 97 subjects completed the study, including 48 subjects in the test food group and 49 subjects in the placebo group. Figure 1 shows the change in sample size over the entire study period as a flow chart. In total, 98 subjects were enrolled, excluding one subject who dropped out just before starting the study and another who disagreed with the data use over the course of the study. Forty-nine subjects in the test group and 49 subjects in the placebo group were subjected to the safety analysis. Later, one subject dropped out due to personal reasons, and five subjects were excluded from the efficacy analysis due to protocol deviations. Consequently, 92 subjects were included in the efficacy analysis, 44 subjects in the test group, and 48 subjects in the placebo group.

Due to deviation from the protocol, two subjects showing Meniere's disease and high blood glucose levels (test food group), one subject with poor compliance (placebo group), and two subjects who were taking multiple medications (test food group) were excluded. For one further subject, we failed to obtain data on body weight, body fat percentage, and BMI because of an Achilles tendon injury at 12 weeks (test food group). 


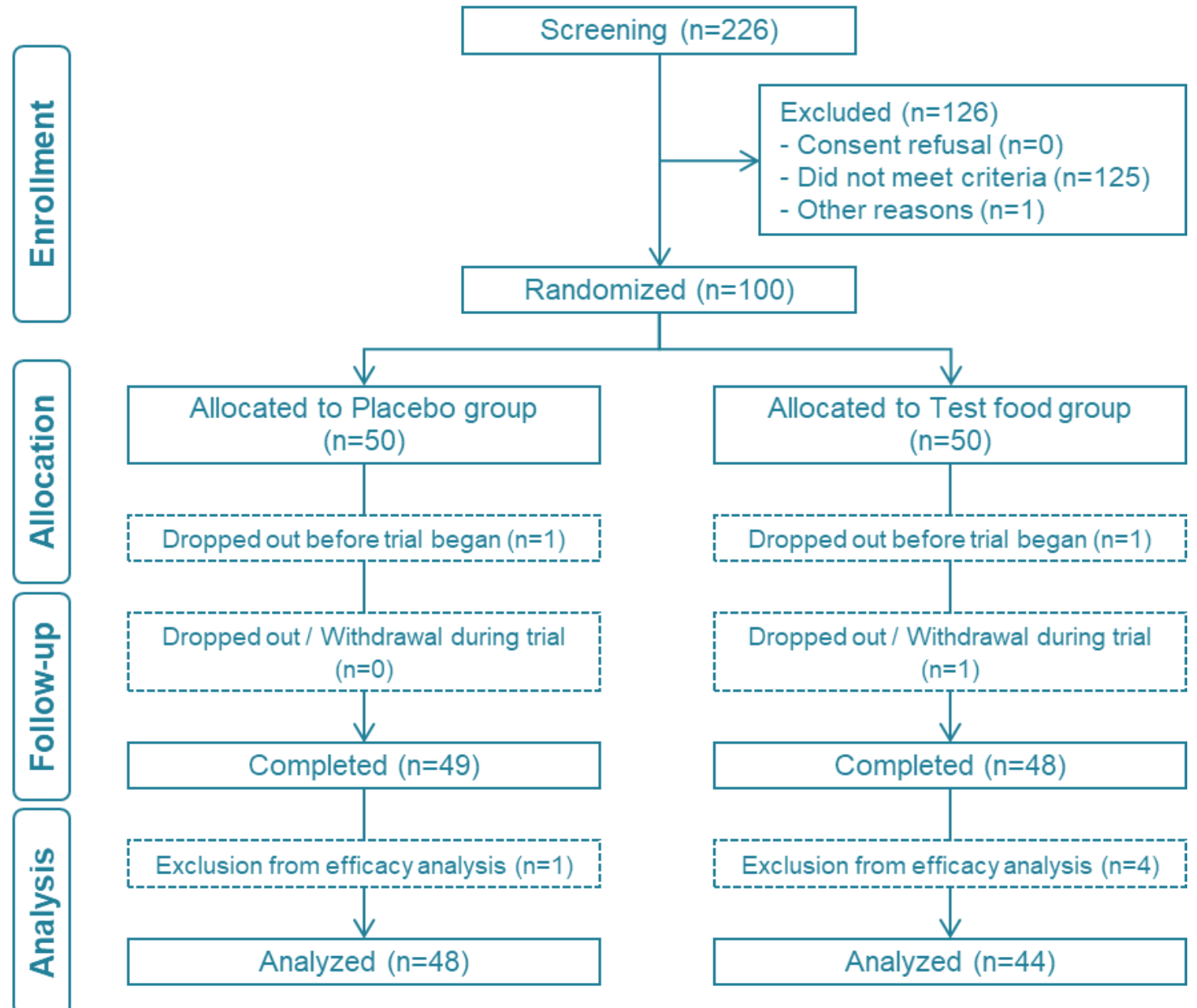

Figure 1. Flowchart of the study.

Table 2 shows the sex, age, BMI, LDL-C, and food intake rate data for the population. There were no significant differences between the two groups, showing that they were adequately allocated into treatment groups.

Table 2. Characteristics of the subjects in the test and placebo food groups.

\begin{tabular}{|c|c|c|c|}
\hline & Placebo $(\mathrm{N}=48)$ & Test food $(N=44)$ & $p$ \\
\hline Male/Female & $18 / 30$ & $16 / 28$ & 1.000 \\
\hline Age (years) & $50.0 \pm 7.7$ & $50.7 \pm 8.1$ & 0.679 \\
\hline BMI $\left(\mathrm{kg} / \mathrm{m}^{2}\right)$ & $25.3 \pm 1.9$ & $25.2 \pm 1.9$ & 0.731 \\
\hline LDL-C (mg/dL) & $120 \pm 11$ & $121 \pm 11$ & 0.679 \\
\hline Intake rate (\%) & $99.1 \pm 1.8$ & $99.4 \pm 1.7$ & 0.196 \\
\hline
\end{tabular}

Values are presented as the mean \pm standard deviation. Independent samples $t$-test was used for age, BMI, LDL-C, Fisher test was used for gender and Mann-Whitney test was used for intake rate.

Evaluation of Laboratory Data: Table 3 shows the effects of the test foods on the serum lipid concentration and body composition. There were no significant changes in LDL-C or any of the other specific variables following consumption of the test food. There were no subjects with fatty livers with L/S ratios of 0.9 or less. 
Table 3. Serum lipid concentration and body composition.

\begin{tabular}{|c|c|c|c|c|c|c|c|}
\hline Variable & & $\mathbf{N}$ & Week 0 & $\Delta$ Week 4 & $\Delta$ Week 8 & $\Delta$ Week 12 & $\begin{array}{l}\text { Test food } \\
\text { effect, } p^{b}\end{array}$ \\
\hline \multirow[t]{3}{*}{ LDL-C (mg/dl) } & Placebo & 48 & $121 \pm 18$ & $0.1 \pm 14.9$ & $-0.3 \pm 14.1$ & $-7.1 \pm 12.3$ & \multirow[t]{3}{*}{0.731} \\
\hline & Test food & 44 & $123 \pm 18$ & $-2.0 \pm 12.8$ & $0.2 \pm 14.7$ & $-3.1 \pm 15.0$ & \\
\hline & $p^{\mathrm{a}}$ & & 0.625 & 0.472 & 0.869 & 0.164 & \\
\hline \multirow[t]{3}{*}{$\mathrm{TC}(\mathrm{mg} / \mathrm{dl})$} & Placebo & 48 & $206 \pm 23$ & $3.1 \pm 18.5$ & $7.0 \pm 17.1$ & $-3.8 \pm 14.7$ & \multirow[t]{3}{*}{0.672} \\
\hline & Test food & 44 & $209 \pm 22$ & $0.0 \pm 16$ & $6.6 \pm 18.6$ & $3.2 \pm 17.0$ & \\
\hline & $p^{\mathrm{a}}$ & & 0.510 & 0.407 & 0.918 & $0.039 *$ & \\
\hline \multirow[t]{3}{*}{ TG (mg/dl) } & Placebo & 48 & $90 \pm 38$ & $11.7 \pm 32.9$ & $14.3 \pm 30.8$ & $3.6 \pm 26.2$ & \multirow[t]{3}{*}{0.440} \\
\hline & Test food & 44 & $91 \pm 36$ & $7.3 \pm 32.6$ & $19.2 \pm 35.5$ & $15.5 \pm 31.7$ & \\
\hline & $p^{\mathrm{a}}$ & & 0.864 & 0.514 & 0.475 & 0.051 & \\
\hline \multirow[t]{3}{*}{ HDL-C (mg/dl) } & Placebo & 48 & $69 \pm 16$ & $-1.0 \pm 5.3$ & $0.5 \pm 5.6$ & $-1.8 \pm 5.6$ & \multirow[t]{3}{*}{0.211} \\
\hline & Test food & 44 & $70 \pm 16$ & $-2.1 \pm 6.4$ & $-1.9 \pm 5.5$ & $-2.0 \pm 5.4$ & \\
\hline & $p^{\mathrm{a}}$ & & 0.729 & 0.371 & $0.043^{*}$ & 0.826 & \\
\hline \multirow[t]{3}{*}{ OxLDL (U/I) } & Placebo & 48 & $108 \pm 31$ & $2.6 \pm 24.9$ & $-6.4 \pm 30.8$ & $26.2 \pm 35.1$ & \multirow[t]{3}{*}{0.286} \\
\hline & Test food & 44 & $108 \pm 34$ & $-5.1 \pm 23.2$ & $-7.0 \pm 27.1$ & $19.2 \pm 30.3$ & \\
\hline & $p^{\mathrm{a}}$ & & 0.922 & 0.125 & 0.923 & 0.312 & \\
\hline \multirow[t]{3}{*}{ BMI $\left(\mathrm{kg} / \mathrm{m}^{2}\right)$} & Placebo & 48 & $25.2 \pm 1.8$ & $-0.1 \pm 0.3$ & $0.0 \pm 0.4$ & $0.0 \pm 0.4$ & \multirow[t]{3}{*}{0.245} \\
\hline & Test food & 44 & $25.0 \pm 1.9$ & $-0.1 \pm 0.4$ & $-0.1 \pm 0.3$ & $-0.1 \pm 0.4$ & \\
\hline & $p^{\mathrm{a}}$ & & 0.631 & 0.398 & 0.124 & 0.417 & \\
\hline \multirow[t]{3}{*}{ L/S ratio } & Placebo & 48 & $2.297 \pm 0.311$ & - & - & $0.025 \pm 0.146$ & \multirow[t]{3}{*}{ - } \\
\hline & Test food & 44 & $2.286 \pm 0.434$ & - & - & $0.012 \pm 0.146$ & \\
\hline & $p^{\mathrm{a}}$ & & 0.887 & - & - & 0.677 & \\
\hline \multirow{3}{*}{$\begin{array}{l}\text { Visceral fat } \\
\text { area }\left(\mathrm{cm}^{2}\right)\end{array}$} & Placebo & 48 & $69.7 \pm 32.3$ & - & - & $1.8 \pm 10.4$ & \multirow[t]{3}{*}{-} \\
\hline & Test food & 44 & $70.7 \pm 41.0$ & - & - & $1.9 \pm 11.9$ & \\
\hline & $p^{\mathrm{a}}$ & & 0.898 & - & - & 0.980 & \\
\hline
\end{tabular}

Values are presented as the mean \pm standard deviation. $p^{\mathrm{a}}$ : Independent samples $t$-tests was performed. $p^{\mathrm{b}}:$ Repeated measures two-way ANOVA was performed. ${ }^{*} p<0.05$ vs. placebo. $\Delta$ Week 4 : changes in values from week 0 to week $4 ; \Delta W e e k$ 8: changes in values from week 0 to week 8; $\Delta$ Week 12: changes in values from week 0 to week 12. 
Table 4 shows the FIB- 4 index and Plt. Changes in the FIB-4 index were lower in the test food group than in the placebo group at 8 and 12 weeks ( $p=0.001, p=0.057)$. The decrease in the platelet count was effectively prevented in the test food group compared with the placebo group at 8 and 12 weeks. The data profile matched well with that of the FIB-4 index.

Table 4. FIB-4 index and Plt.

\begin{tabular}{|c|c|c|c|c|c|c|c|}
\hline Variable & & $\mathbf{N}$ & Week 0 & $\Delta$ Week 4 & $\Delta$ Week 8 & $\Delta$ Week 12 & $\begin{array}{l}\text { Test food } \\
\text { effect, } p^{b}\end{array}$ \\
\hline \multirow[t]{3}{*}{ FIB-4 index } & Placebo & 48 & $0.89 \pm 0.28$ & $0.00 \pm 0.11$ & $0.06 \pm 0.10$ & $0.02 \pm 0.13$ & \multirow[t]{3}{*}{$0.029 *$} \\
\hline & Test food & 44 & $1.01 \pm 0.43$ & $0.00 \pm 0.13$ & $-0.03 \pm 0.14$ & $-0.04 \pm 0.18$ & \\
\hline & $p^{\mathrm{a}}$ & & 0.118 & 0.903 & $0.001 * *$ & 0.057 & \\
\hline \multirow[t]{3}{*}{$\operatorname{Plt}\left(10^{4} / \mu \mathrm{L}\right)$} & Placebo & 48 & $27.2 \pm 4.9$ & $-0.6 \pm 2.3$ & $-1.1 \pm 2.0$ & $-0.7 \pm 2.0$ & \multirow[t]{3}{*}{$0.014^{*}$} \\
\hline & Test food & 44 & $25.9 \pm 5.3$ & $-0.3 \pm 2.1$ & $0.4 \pm 1.8$ & $0.4 \pm 2.6$ & \\
\hline & $p^{a}$ & & 0.207 & 0.480 & $<0.001^{* *}$ & $0.031 *$ & \\
\hline
\end{tabular}

Values are presented as the mean \pm standard deviation. $p^{\text {a }}$ : Independent samples $t$-tests was performed. $p^{\text {b: }}$ Repeated measures two-way ANOVA was performed. $* p<0.05$ vs. placebo. $\Delta$ Week 4 : changes in values from week 0 to week $4 ; \Delta$ Week 8 : changes in values from week 0 to week 8 ; $\Delta$ Week 12: changes in values from week 0 to week 12 .

Next, we subdivided the subjects into those with a BMI below $25 \mathrm{~kg} / \mathrm{m}^{2}$ and those with a BMI of $25 \mathrm{~kg} / \mathrm{m}^{2}$ and higher and carried out a subgroup analysis. We have confirmed no significant differences in gender, age, BMI, LDL-C, and consumption rates for each subgroup population.

The BMI subgroup analysis showed a significant reduction in the change in oxLDL at week 12 in subjects with a BMI of $25 \mathrm{~kg} / \mathrm{m}^{2}$ or higher compared to the placebo group ( $p=0.009$ ). No significant differences were observed in those with a BMI below $25 \mathrm{~kg} / \mathrm{m}^{2}$ (Tables 5 ). There were no significant differences between the test food group and the placebo group in participants with a BMI of $25 \mathrm{~kg} / \mathrm{m}^{2}$ or higher in the other blood lipids (LDL-C, TC, HDL-C, and TGs), body weight, BMI, L/S ratio, visceral fat area, total abdominal fat area, or abdominal subcutaneous fat area.

Table 5. oxLDL in the subgroups.

\begin{tabular}{|c|c|c|c|c|c|c|c|c|}
\hline Variable & Subgroup & & $\mathbf{N}$ & Week 0 & $\Delta$ Week 4 & $\Delta$ Week 8 & $\Delta$ Week 12 & $\begin{array}{l}\text { Test food } \\
\text { effect, } p^{b}\end{array}$ \\
\hline \multirow[t]{6}{*}{$\operatorname{oxLD}(U / I)$} & BMI & Placebo & 27 & $107 \pm 31$ & $-0.4 \pm 23.8$ & $-13.6 \pm 26.8$ & $17.9 \pm 33.4$ & \multirow[t]{3}{*}{0.251} \\
\hline & \multirow[t]{2}{*}{$<25 \mathrm{~kg} / \mathrm{m}^{2}$} & Test food & 22 & $97 \pm 26$ & $-3.4 \pm 18.9$ & $-1.6 \pm 23.4$ & $29.2 \pm 26.8$ & \\
\hline & & $p^{\mathrm{a}}$ & & 0.270 & 0.637 & 0.107 & 0.207 & \\
\hline & BMI & Placebo & 21 & $109 \pm 32$ & $6.6 \pm 26.2$ & $2.8 \pm 33.7$ & $36.9 \pm 35.3$ & \multirow[t]{3}{*}{$0.015^{*}$} \\
\hline & \multirow[t]{2}{*}{$\geq 25 \mathrm{~kg} / \mathrm{m}^{2}$} & Test food & 22 & $120 \pm 37$ & $-6.9 \pm 27.1$ & $-12.4 \pm 30.0$ & $9.3 \pm 30.8$ & \\
\hline & & $p^{\mathrm{a}}$ & & 0.340 & 0.105 & 0.126 & $0.009 * *$ & \\
\hline \multirow[t]{3}{*}{$\operatorname{oxLDL}(\mathrm{U} / \mathrm{I})$} & \multirow[t]{3}{*}{ All Case } & Placebo & 48 & $108 \pm 31$ & $2.6 \pm 24.9$ & $-6.4 \pm 30.8$ & $26.2 \pm 35.1$ & \multirow[t]{3}{*}{0.286} \\
\hline & & Test food & 44 & $108 \pm 34$ & $-5.1 \pm 23.2$ & $-7.0 \pm 27.1$ & $19.2 \pm 30.3$ & \\
\hline & & $p^{a}$ & & 0.922 & 0.125 & 0.923 & 0.312 & \\
\hline
\end{tabular}

Values are presented as the mean \pm standard deviation. $p^{\text {a }}$ : Independent samples $t$-tests was performed. $p^{\text {b: }}$ Repeated measures two-way ANOVA was performed. ${ }^{*} p<0.05$ vs. placebo ${ }^{* *} p<0.01$ vs. placebo. $\Delta$ Week 4 : changes in values from week 0 to week 4 ; $\Delta$ Week 8: changes in values from week 0 to week $8 ; \Delta$ Week 12: changes in values from week 0 to week 12. 
Additional Analysis: Since we found that the increase in oxLDL was suppressed in subjects with a BMI of $25 \mathrm{~kg} / \mathrm{m}^{2}$ or higher in the test food group, we conducted further analyses to investigate what factors contributed to the oxLDL increase. Using the initial values (week 0 ) of the respective variables of 92 subjects determined in the efficacy analysis, Pearson correlation coefficients of the variables that were highly correlated with oxLDL were analyzed and extracted (Table 6). OxLDL was positively correlated with LDL-C, TG, and visceral fat and inversely correlated with HDL-C. OxLDL was also associated with hematologic indices and was positively correlated with WBC, RBC, $\mathrm{Hb}$, and $\mathrm{Ht}$.

Table 6. Correlations between oxLDL and variables.

\begin{tabular}{|lllllll|}
\hline Variable & LDL-C & HDL-C & TG & HbA1c & Visceral fat area & L/S ratio \\
\hline Pearson correlation coefficient & $0.414^{* *}$ & $-0.318^{* *}$ & $0.233^{*}$ & 0.140 & $0.359^{* *}$ & -0.156 \\
\hline $\boldsymbol{p}$ & 0.000 & 0.002 & 0.026 & 0.182 & 0.000 & 0.139 \\
\hline $\mathbf{N}$ & 92 & 92 & 92 & 92 & 92 & 92 \\
\hline
\end{tabular}

\begin{tabular}{|l|llll|}
\hline Variable & WBC & RBC & Hb & Ht \\
\hline Pearson correlation coefficient & $0.287^{*}$ & $0.379^{* *}$ & $0.302^{* *}$ & $0.306^{* *}$ \\
\hline $\boldsymbol{p}$ & 0.006 & 0.000 & 0.003 & 0.003 \\
\hline $\mathbf{N}$ & 92 & 92 & 92 & 92 \\
\hline
\end{tabular}

The correlation significance of two variables was tested using Pearson's correlation coefficient.

$* p<0.05, * * p<0.01$.

When we examined the correlations between the FIB-4 index change ( $\Delta 12$ week) and the initial value of each variable, we found a significant correlation with oxLDL and a positive correlation with visceral fat (Table 7). There were no correlations with LDL-C, TGs, or HbA1c.

Table 7. Correlations between the FIB- 4 index change ( $\Delta 12$ week) and variables.

\begin{tabular}{|llllll|}
\hline Variable & Visceral fat areas & OxLDL & LDL & TG & HbA1c \\
\hline Pearson correlation coefficient & 0.121 & $0.275^{* *}$ & 0.011 & 0.116 & -0.073 \\
\hline $\boldsymbol{p}$ & 0.252 & 0.008 & 0.917 & 0.272 & 9.489 \\
\hline $\mathbf{N}$ & 92 & 92 & 92 & 92 \\
\hline
\end{tabular}

The correlation significance of two variables was tested using Pearson's correlation coefficient

$* p<0.05,{ }^{* *} p<0.01$ 
Assessment of Safety: We found no significant differences in adverse events between the two groups. The number of adverse events in the test food group was 42 (35 subjects), whereas there were 49 events in the placebo group (38 subjects). There were no serious adverse events. None of the adverse events were related to the study food. Therefore, we concluded that no adverse reactions were present in this study.

We listed minimal changes between the two groups at any time point as follows. In the test diet group, there were three events of gastrointestinal symptoms for three subjects. Other events included malaise in one subject, facial rash in one subject, urticaria in one subject, and heat stroke in one subject. Headache, nausea, vomiting, chills, cold sweat, decreased appetite, stiff shoulder, edema of the lower limbs, stomach pain, and allergy (cough) were experienced by one subject. Nasal discharge occurred in one subject, abnormal bleeding in one subject, chest distress in one subject, and Achilles' tendon rupture in one subject. These events were confirmed to be unrelated to the test food by a physician.

As for the placebo group, there were four events of gastrointestinal symptoms (abdominal pain, diarrhea, and

\section{DISCUSSION}

The test food, burdock extract containing arctigenin, suppressed the increase in oxLDL after 12 weeks in subjects with a BMI of $25 \mathrm{~kg} / \mathrm{m}^{2}$ or higher. Subjects with a BMI higher than $25 \mathrm{~kg} / \mathrm{m}^{2}$ often have larger visceral fat areas than subjects with lower BMI values. It is expected that oxLDL is more likely to be generated in subjects with a high level of visceral fat. It is hypothesized that increased oxLDL in the visceral fat might be specifically suppressed by the arctigenin in the test food. We also observed a significant positive association between oxLDL and the visceral fat area measured by CT scans (data not shown). This indicates that oxLDL production is regulated by arctigenin through the suppression of reactive oxygen species (ROS) production and inflammatory factors in visceral fat.

Abdominal obesity is a principal causative factor for the development of metabolic syndrome. Furukawa et al. gastralgia) in three subjects. Other events included dentalrelated complaints, including periodontal disease, dental caries, and alveolar pyorrhea (three events in three subjects), herpes labialis (two events in one subject), bronchopneumonia (one event in one subject), knee pain (one event in one subject), left shoulder pain (one event in one subject), myalgia (one event in one subject), burn (one event in one subject), and conjunctivitis (one event in one subject). These events were confirmed to be unrelated to the placebo by a physician.

For the laboratory data, we measured in terms of hematology parameters (WBC, RBC, $\mathrm{Hb}, \mathrm{Ht}, \mathrm{Plt}, \mathrm{MCH}$, MCHC, and MCV), hepatic function (AST, ALT, $Y-G T P, A L P$, $\mathrm{LDH}, \mathrm{T}-\mathrm{Bil}$ and $\mathrm{Alb}$ ), renal function (BUN, CRE, and UA), and blood glucose (fasting blood glucose and $\mathrm{HbA} 1 \mathrm{c}$ ). Statistical analyses revealed significant differences between the two groups in terms of WBC, Ht, PIt and Alb. However, all of these changes were within the normal range and were not clinically significant (data not shown). No significant differences were found regarding vital signs (BP and pulse rate at each visit) or the urinalysis $(\mathrm{pH}$, glucose, protein, occult blood, urobilinogen, ketone bodies, urinalysis) between the two groups (data not shown).

reported that oxidative stress in accumulated fat is an important pathogenic mechanism involved in the development of obesity-associated metabolic syndrome. Fat accumulation in the abdomen is strongly correlated with systemic oxidative stress in humans. The production of ROS was shown to increase selectively in the adipose tissue of obese mice [19]. In abdominal adipocytes, the expression of NADPH oxidase, a ROS-producing enzyme, is elevated, while ROS production is elevated due to the decreased expression of antioxidant enzymes, such as superoxide dismutase (SOD). Wu et al. reported that arctigenin reduced ROS production in the rat L6 skeletal muscle cell line and demonstrated that arctigenin efficiently enhances rats' swimming endurance by elevation of the antioxidant capacity of the skeletal muscles [20]. Thus, the antioxidative action of arctigenin in the test food may prevent LDL oxidation by inhibiting 
ROS production. In addition, the inflammasome-mediated anti-inflammatory effects of arctigenin have been reported [21-23]. In brief, arctigenin was found to attenuate ischemic stroke by effectively inhibiting cerebral-ischemia-induced Nod-like receptor protein 3 (NLRP3) inflammasome activation through the SIRT1 pathway [21]. Based on this mechanism, arctigenin might attenuate inflammation in dextran-sulfate-induced acute colitis [22] and colitis-associated cancer [23]. In the abdominal adipose tissue, fatty acids may function as endogenous pro-inflammatory agents, which leads to the production of macrophage-releasing inflammatory cytokines and furthermore involvement of Toll-like receptor 4 (TLR-4), a pattern-recognition receptor of the innate immune system [24]. When TLR-4 stimulates NLRP3 inflammasome activation, mitochondria are damaged, generating reactive oxygen species, which oxidize mitochondrial DNA. This oxidized mitochondrial DNA activates the NLRP3 inflammasome by translocating to the cytosol and binding to NLRP3 [25]. Reactive oxygen species production promotes LDL-C oxidation and extracellular catabolism of LDL aggregates [26], suggesting that TLR4 activation in adipose tissue is associated with LDL-C oxidation. It is of interest that arctigenin prevents inflammatory progression by suppressing NLRP3 activation and fatty acid oxidation metabolism in macrophages [23].

Arctigenin is a potent activator of AMPK via the inhibition of respiratory complex $\mathrm{I}$, and it has beneficial effects on metabolic disorders in mice [27]. This highlights the potential value of arctigenin for the treatment of type 2 diabetes. Activation of AMPK stimulates SIRT1, which may reduce the accumulation of oxLDL via the autophagylysosomal pathway by SIRT1 in vascular endothelial cells [28]. Metformin, a typical compound with AMPKactivating action, suppresses oxLDL generation and lectinlike oxidized low-density lipoprotein receptor 1 (LOX-1) expression in vascular endothelial cells, and the activation of AMPK and SIRT1 contributes to a mechanistic pathway [29]. Thus, arctigenin may inhibit the activation of NLRP3 inflammasomes and fatty acid oxidation in adipose tissue and reduce oxLDL via the SIRT1-mediated autophagylysosomal pathway through AMPK activation. Furthermore, it has been reported that oxLDL promotes TLR4 expression in macrophages [30], and the TLR4/NFKB signaling system is involved in LOX1 upregulation by oxLDL [31]. These findings suggest that TLR4 activation and LDL oxidation in adipose tissue may exert various effects on the homeostasis of the total body beyond arteriosclerosis.

In the present study, we demonstrated a correlation between oxLDL and changes in the FIB-4 index (Table 9), suggesting that oxLDL is a risk factor for hepatic fibrosis. Hepatic fibrosis is often difficult to detect at the early stage, because it progresses without any specific symptoms. In this context, the FIB-4 index is a useful method for monitoring hepatic fibrosis stages using age, AST and ALT concentrations, and platelet counts. Using this practical method, Sumida et al. assessed the degree of hepatic fibrosis progression in subjects with nonalcoholic fatty liver disease (NAFLD). They found that the FIB-4 index is a suitable scoring system for identifying hepatic fibrosis in Japanese people [18]. In our study, none of the healthy subjects had a fatty liver L/S ratio of less than 0.9 , but we found that the FIB-4 index changed following supplementation with the test food containing arctigenin. When correlations between changes in the FIB-4 index and the oxLDL concentration or visceral fat area were analyzed in the test and placebo groups, we identified significant differences in response between the two groups (Figure 2 ). This indicates the possibility that oxLDL is correlated with the increase in the FIB-4 index, indicating that the test food may prevent the elevation of the FIB- 4 index by controlling the oxidation of LDL. The correlation between the visceral fat area and FIB-4 index change was positive in the placebo-treated group, while the correlation disappeared in the test food group, which is consistent with the reduction in the FIB-4 index shown in this group. The test food might prevent an increase in the FIB-4 index by controlling LDL oxidation, potentially by controlling inflammatory factors released from visceral fat tissue. Placebo and test meals were compared by scatter diagram. 

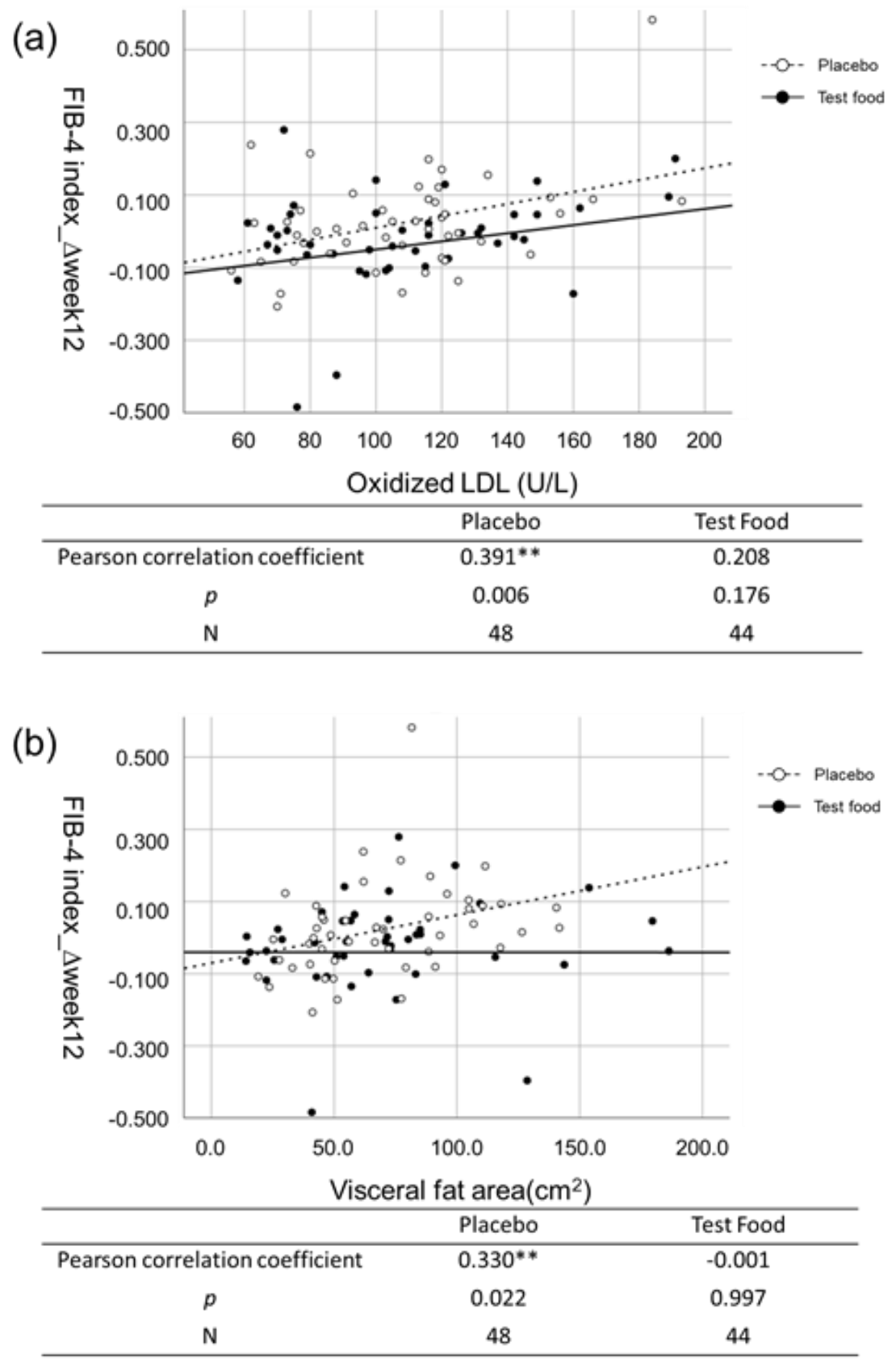

Figure 2. Correlations between FIB-4 index change and (a) the initial oxLDL value or (b) the initial visceral fat area in the placebo and test food groups. The correlation significance of two variables was tested using Pearson's correlation coefficient. $* * p<0.01$

These results suggest that suppression of the increase in the FIB-4 index indicates to the suppression of hepatic fibrosis progression. For hepatic fibrosis, Seki et al. reported that the activation of TLR4 enhances the sensitivity to TGF $\beta$ by reducing downstream transforming growth factor $\beta$ (TGF $\beta$ ) pseudoreceptor Bambi expression in hepatic stellate cells, which results in the progression of liver fibrosis [32]. In early-stage arteriosclerotic foci, platelet-derived growth factor (PDGF) is released by platelets and macrophages recruited in the injured arteries' intima during the early arteriosclerotic stage. Platelets play a critical role in inducing the migration to and proliferation of smooth muscle cells in the intima [33]. PDGF is also known to facilitate the progression of hepatic 
fibrosis through the pathway, modulating a broad spectrum of cellular processes, including cell growth, differentiation, and inflammation [34]. It is conceivable that excessive oxLDL may lead to hepatic fibrosis via TGF $\beta$ and PDGF.

The platelet count is known to decrease due to hepatic dysfunction and fibrosis. Possible causes of this thrombocytopenia include increased destruction of platelets pooling in the enlarged spleen [35], and decreased activity of the hematopoietic growth factor thrombopoietin in liver [36]. Recently, it became clear that platelets play a variety of important roles, not only in terms of their physiological functions in coagulation and hemostasis, but also in tissue repair and angiogenesis. An example would be how platelet-derived serotonin has been suggested to be involved in the initiation of liver regeneration [37], while platelet-rich plasma (PRP) is currently used in various medical fields such as tissue regeneration and wound healing. [38]. Nam et al. reported that arctigenin attenuated platelet activation and clot retraction through the regulation of thromboxane $A 2$ synthesis and the cAMP pathway as well as maintaining the platelet count balance [39]. Barreto et al. reported that LOX-1 is also expressed in platelets, where it enhances platelet activation, adhesion to endothelial cells, and ADP-mediated aggregation, thereby favoring thrombus formation [40]. It is possible that suppression of oxLDL contributes to the maintenance of platelet count. In the present study, we demonstrated correlations between the initial values and changes at week 12 for red blood cell and platelet counts and showed that their increases were more robust in the test food group (Figures 3 and 4). This indicates that the consumption of the test foods containing arctigenin increased red blood cell and platelet counts in the lower value population. It is hypothesized that this blood homeostasis mechanism may suppress increases in the FIB-4 index. These results suggest that the maintenance of platelet count and the increased inhibitory effect of the FIB-4 index may be outcomes related to the maintenance of blood flow and hepatic function associated with consumption of the test food.

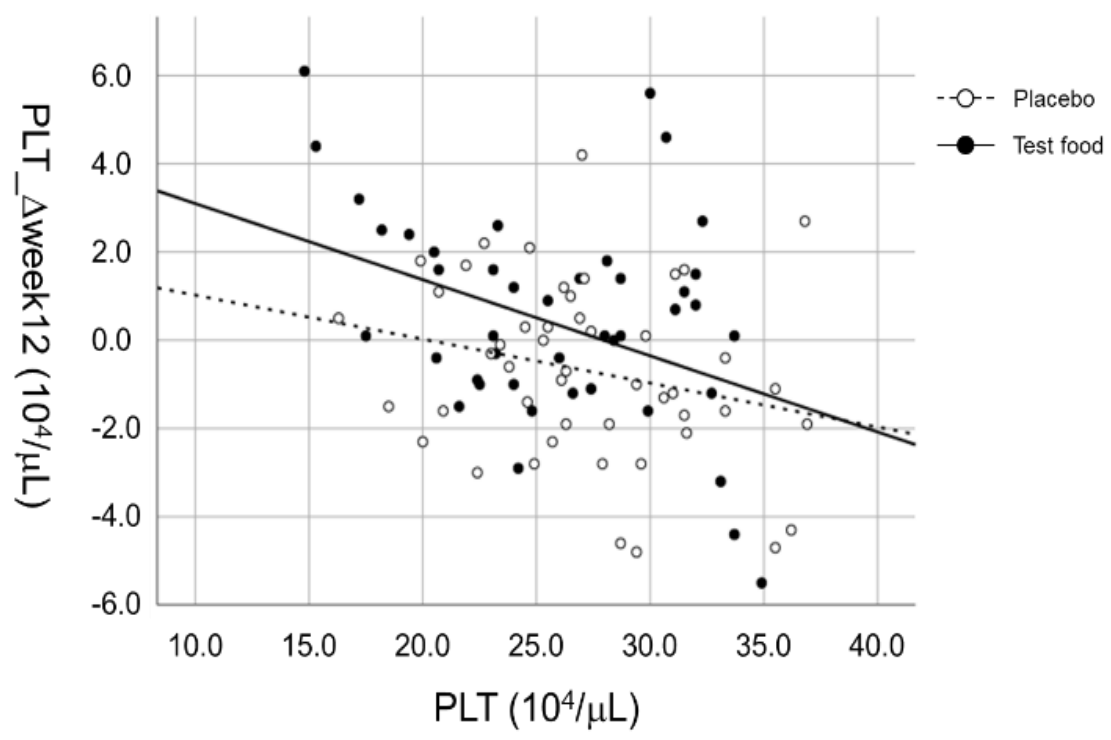

Figure 3. Correlation between initial Plt counts and changes at 12 weeks in the placebo and test food groups. 


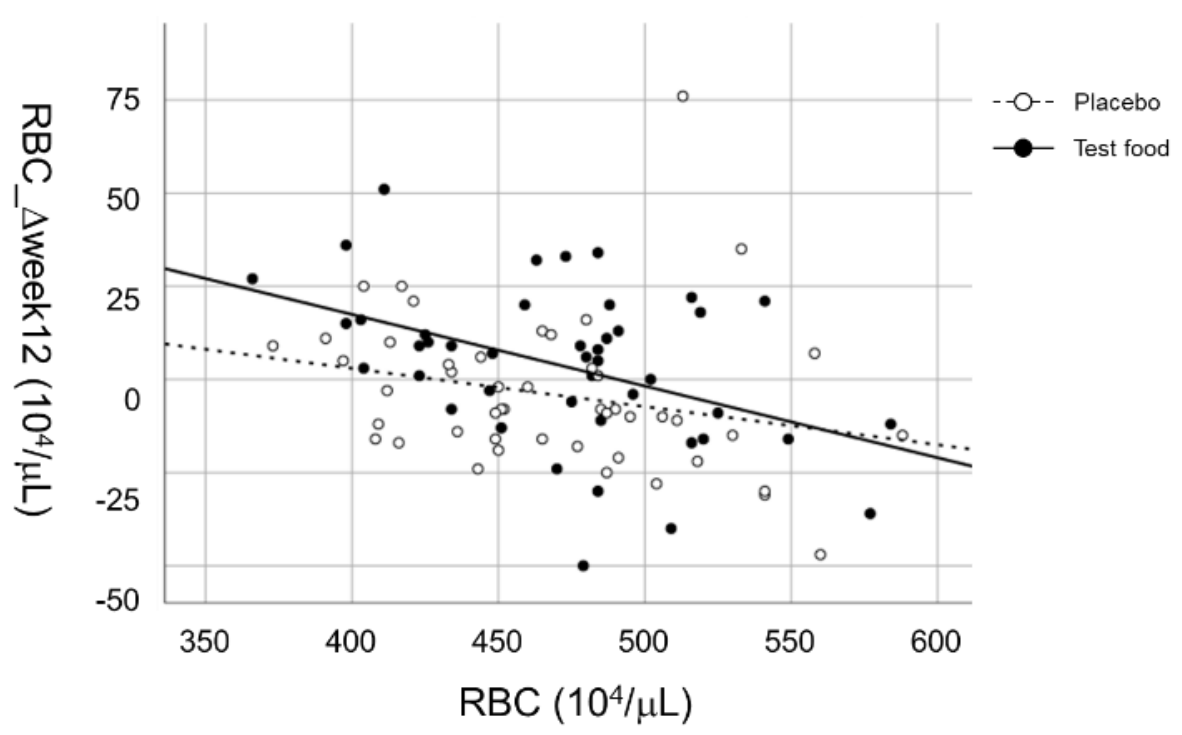

Figure 4. Correlations between initial RBC values and changes at 12 weeks in the placebo and test food groups.

Taken together, these results suggest that the test food containing burdock extract rich in arctigenin primarily reduces oxLDL and maintains factors related to

\section{CONCLUSION}

In a study involving 12 weeks of the supplementation of healthy volunteers with a test food containing burdock extract rich in arctigenin, we demonstrated that the test food significantly inhibited an increase in oxLDL in subjects with a BMI of $25 \mathrm{~kg} / \mathrm{m}^{2}$ or higher. Since the test food is shown to inhibit an increase in the FIB-4 index, we anticipate that it may effectively inhibit the progression of hepatic fibrosis as well as arteriosclerosis, as these conditions are primarily caused by LDL oxidation. Throughout this clinical trial, we found no adverse effects attributable to the test food, which indicates that the food safety and food efficacy are acceptable.

List of abbreviations: LDL-C: low-density lipoprotein cholesterol, oxLDL: oxidized LDL, MDA-LDL: malondialdehyde-induced apo-B, PWV: pulse-wave velocity, NAFLD: nonalcoholic fatty liver disease, FIB-4 index: liver fibrosis index, AMPK: AMP-activated protein kinase, ACC: acetyl CoA carboxylase, PPARy: peroxisome proliferator-activated receptor $\gamma$, SREBP1c: sterol regulatory element-binding transcription factor $1, \mathrm{BMI}$ : body mass index, L/S ratio: ratio of liver to spleen, UMIN: hematological homeostasis, such as the platelet count, which is beneficial for the prevention of hepatic fibrosis.

University Hospital Medical Information Network, CT: computed tomography, TC: total cholesterol, TG: triglyceride, HDL-C: HDL-cholesterol, BP: blood pressure, WBC: white blood cell, RBC: red blood cell, $\mathrm{Hb}$ : hemoglobin, $\mathrm{Ht}$ : hematocrit, Plt: platelets, $\mathrm{MCH}$ : mean corpuscular hemoglobin, MCHC; mean corpuscular hemoglobin concentration, MCV: mean corpuscular volume, AST: aspartate aminotransferase, ALT: alanine aminotransferase, GTP: $\gamma$-glutamic pyruvic transaminase, ALP: alkaline phosphatase, LDH: lactate dehydrogenase, TBil: total bilirubin, Alb: albumin, BUN: blood urea nitrogen, CRE: creatinine, UA; uric acid, HbA1c: hemoglobin A1c, ROS: reactive oxygen species, SOD: superoxide dismutase, NLRP3: Nod-like receptor protein 3, TLR-4: Toll-like receptor 4, LOX-1: lectin-like oxidized low-density lipoprotein receptor 1 , TGF $\beta$ : transforming growth factor $\beta$, PDGF: platelet-derived growth factor, PRP: platelet-rich plasma

Competing interests: This study was conducted with research funds under contract with Kracie Holdings, Ltd. Under the "Hokkaido Information University Bioethics Committee Regulations," the research director and the 
investigator of this study declared necessary conflicts of interest to the ethical review committee.

Authors' contributions: Conceptualization, N.I., A.O., J.N.; methodology, A.O., J.N.; software, H.H.; validation, A.O., S.Y.,S.I., J.N.; formal analysis, A.O., S.Y.,S.I., J.N.; investigation, N.I., A.O. J.N.; resources, A.O. S.Y.,S.I.; data curation, N.I., H.H., A.O.; writing-original draft preparation, N.I., A.O., S.Y., J.N.; writing-review and editing, N.I., A.O., S.Y., S.I., H.H., J.N.; visualization, A.O.,

\section{REFERENCES}

1. Tsimikas S: Oxidized low-density lipoprotein biomarkers in atherosclerosis. Curr. Atheroscler. Rep. 2006, 8:55-61.

2. Kokubo Y, Kohmo K, Higashiyama A, Watanabe M. Miyamoto Y: 56th General Meeting of the Japanese Society of Hepatology Joint Session of the Japanese Society of Hepatology-Japanese Society of Arteriosclerosis Nonalcoholic Fatty Liver Disease and Dyslipidemia, Liver 2020, 61 suppl., A301.

3. He Y, Fan Q, Cai T, Huang W, Xie X, Wen Y, Shi Z: Molecular Mechanisms of the Action of Arctigenin in Cancer. Biomed. Pharmacother. 2018, 108:403-407.

4. Ferracane R, Graziani G, Gallo M, Fogliano V, Ritieni A: Metabolic Profile of the Bioactive Compounds of Burdock (Arctium Lappa) Seeds, Roots and Leaves. J. Biomed. Anal. 2010, 51:399-404.

5. Liu S, Chen K, Schliemann W, Strack D: Isolation and Identification of Arctiin and Arctigenin in Leaves of Burdock (Arctium Lappa L.) by Polyamide Column Chromatography in Combination with Hplc-Esi/Ms. Phytochem. Anal. 2005, 16:8689.

6. Nose M, Fujimoto T, Takeda T, Nishibe S, Ogihara Y: Structural transformation of lignan compounds in rat gastrointestinal tract. Planta Med. 1992, 58:520-523.

7. Yang J, Yin,HS, Cao YJ, Jiang ZA, Li YJ, Song MC, Wang YF, Wang $\mathrm{ZH}$, Yang $\mathrm{R}$, Jiang $\mathrm{YF}$, et al.: Arctigenin Attenuates Ischemia/Reperfusion Induced Ventricular Arrhythmias by Decreasing Oxidative Stress in Rats. Cell. Physiol. Biochem. 2018, 49:728-742.

8. Predes FS, Ruiz AL, Carvalho JE, Foglio MA, Dolder $\mathrm{H}$ : Antioxidative and in Vitro Antiproliferative Activity of Arctium Lappa Root Extracts. BMC Complement. Altern. Med. 2011,11:25.

9. Kang HS, Lee JY, Kim CJ: Anti-Inflammatory Activity of Arctigenin from Forsythiae Fructus. J. Ethnopharmacol. 2008,116:305-312.
S.Y., H.H.; supervision, S.I., J.N.; project administration, S.I., J.N.; funding acquisition, S.I., J.N. All authors have read and agreed to the published version of the manuscript.

Acknowledgments and funding: We are deeply grateful to members of the Hokkaido Information University, Center of Health Information Science (T. Saito, and Y. Fukuda) for their management and assistance with the clinical trial. Kracie Holdings, Ltd. covered the research costs and provided the test foods for this study.

10. Lee JY, Kim CJ: Arctigenin, a Phenylpropanoid Dibenzylbutyrolactone Lignan, Inhibits Type I-Iv Allergic Inflammation and Pro-Inflammatory Enzymes. Arch. Pharm. Res. 2010, 33:947-957.

11. Kim Y, Hollenbaugh JA, Kim DH, Kim B: Novel Pi3k/Akt Inhibitors Screened by the Cytoprotective Function of Human Immunodeficiency Virus Type 1 Tat. PLoS One 2011, 6:e21781.

12. Tsai WJ., Chang CT, Wang GJ, Lee TH, Chang SF, Lu SC, Kuo YC: Arctigenin from Arctium Lappa Inhibits Interleukin-2 and Interferon Gene Expression in Primary Human T Lymphocytes. Chin. Med. 2011, 6:12.

13. Song $Y$, Li $X$, Liu $Y$, Hu Y, Yang R: Arctigenin Improves Lipid Metabolism by Regulating AMP-Activated Protein Kinase and Downstream Signaling Pathways. J. Cell. Biochem. 2019, 120:13275-13288.

14. Han YH, Kee JY, Park J, Kim HL, Jeong MY, Kim DS, Jeon YD, Jung Y, Youn DH, Kang JW, et al.: Arctigenin Inhibits Adipogenesis by Inducing Ampk Activation and Reduces Weight Gain in High-Fat Diet-Induced Obese Mice. J. Cell. Biochem. 2016, 117:20672077.

15. Watanabe S, Ohno A, Yomoda S, Inamasu S: Anti-obesity effects of burdock sprout extract in high fat diet-induced obesity in mice. The 2018 Annual Meeting of Japan Society for Bioscience, Biotechnology, and Agrochemistry 2018:3B02a14. https://jsbba.bioweb.ne.jp/jsbba_db/index.html

16. Sterling RK, Lissen E Clumeck N, Sola R, Correa MC, Montaner J, S Sulkowski M, Torriani FJ, Dieterich DT, Thomas DL, et al.: Development of a simple noninvasive index to predict significant fibrosis in patients with HIV/HCV coinfection. Hepatology 2006, 43:1317-1325.

17. Shah AG, Lydecker A, Murray K, Tetri BN, Contos MJ, Sanyal AJ: Nash Clinical Research Network. Comparison of noninvasive 
markers of fibrosis in patients with nonalcoholic fatty liver disease. Clin. Gastroenterol. Hepatol. 2009, 7:1104-1112.

18. Sumida $Y$, Yoneda $M$, Hyogo $H$, Itoh $Y$, Ono $M$, Fujii $H$, Eguchi $Y$, Suzuki Y, Aoki N, Kanemasa K, et al.: Validation of the Fib4 index in a Japanese nonalcoholic fatty liver disease population. BMC Gastroenterol. 2012, 12:2.

19. Furukawa S, Fujita T, Shimabukuro M, Iwaki M, Yamada $Y$, Nakajima Y, Nakayama O, Makishima M, Matsuda M, Shimomura I: Increased oxidative stress in obesity and its impact on metabolic syndrome. J. Clin. Invest. 2004, 114:1752-1761.

20. Wu RM, Sun YY, Zhou TT, Zhu ZY, Zhuang JJ, Tang X, Chen J, Hu $\mathrm{LH}$, Shen X: Arctigenin enhances swimming endurance of sedentary rats partially by regulation of antioxidant pathways. Acta Pharmacol. Sin. 2014, 35:1274-1284.

21. Zhang S, Jiang L, Che F, Lu Y, Xie Z, Wang H: Arctigenin attenuates ischemic stroke via SIRT1-dependent inhibition of NLRP3 inflammasome. Biochem. Biophys. Res. Commun. 2017, 493:821-826

22. Pu Z, Han C, Zhang W, Xu M, Wu Z, Liu Y, Wu M, Sun H, Xie H: Systematic understanding of the mechanism and effects of Arctigenin attenuates inflammation in dextran sulfate sodiuminduced acute colitis through suppression of NLRP3 inflammasome by SIRT1. Am. J. Transl. Res. 2019, 11:3992-4009.

23. Qiao S, Lv C, Tao Y, Miao Y, Zhu Y, Zhang W, Sun D, Yun X, Xia Y, Wei Z, Dai Y: Arctigenin disrupts NLRP3 inflammasome assembly in colonic macrophages via downregulating fatty acid oxidation to prevent colitis-associated cancer. Cancer Lett. 2020, 491:162179.

24. Suganami T, Tanimoto-Koyama K, Nishida J, Itoh M, Yuan X, Mizuarai S, Kotani H, Yamaoka S, Miyake K, Aoe S, et al.: Role of the Toll-like receptor 4/NF-kappaB pathway in saturated fatty acid-induced inflammatory changes in the interaction between adipocytes and macrophages. Arterioscler. Thromb. Vasc. Biol. 2007, 27:84-91.

25. Shimada K, Crother TR, Karlin J, Dagvadorj J, Chiba N, Chen S, Ramanujan VK, Wolf AJ, Vergnes L, Ojcius, D.M, et al.: Oxidized mitochondrial DNA activates the NLRP3 inflammasome during apoptosis. Immunity 2012, 36:401-414.

26. Singh RK, Haka AS, Asmal A, Barbosa-Lorenzi VC, Grosheva I, Chin HF, Xiong Y, Hla T, Maxfield FR: TLR4 (Toll-Like Receptor 4)Dependent Signaling Drives Extracellular Catabolism of LDL (Low-Density Lipoprotein) Aggregates. Arterioscler. Thromb. Vasc. Biol. 2020, 40:86-102.

27. Huang SL, Yu RT, Gong J, Feng Y, Dai YL, Hu F, Hu YH, Tao YD, Y Leng $\mathrm{Y}$ : Arctigenin, a natural compound, activates AMPactivated protein kinase via inhibition of mitochondria complex
I and ameliorates metabolic disorders in ob/ob mice. Diabetologia 2012, 55:1469-1481.

28. Zhang Y, Sun J, Yu X, Shi L, Du W, Hu L, Liu C, Cao Y: SIRT1 regulates accumulation of oxidized LDL in HUVEC via the autophagy-lysosomal pathway. Prostaglandins Other Lipid Mediat. 2016, 22:37-44.

29. Hung $\mathrm{CH}$, Chan SH, Chu PM, Lin HC, Tsai KL: Metformin regulates oxLDL-facilitated endothelial dysfunction by modulation of SIRT1 through repressing LOX-1-modulated oxidative signaling. Oncotarget 2016, 7:10774-10787.

30. Howell KW, Meng X, Fullerton DA, Jin C, Reece TB, Cleveland Jr JC: Toll-like receptor 4 mediates oxidized LDL-induced macrophage differentiation to foam cells. J. Surg. Res. 2011, 171:e27-31.

31. Feng Y, Cai ZR, Tang Y, Hu G, Lu J, He D, Wang S: TLR4/NF- k B signaling pathway-mediated and oxLDL-induced up- regulation of LOX-1, MCP-1, and VCAM-1 expressions in human umbilical vein endothelial cells. Genet. Mol. Res. 2014, 13:680-695.

32. Seki E, De Minicis S, Osterreicher $\mathrm{CH}$, Kluwe J, Osawa Y, Brenner DA, Schwabe RF: TLR4 enhances TGF-beta signaling and hepatic fibrosis. Nat. Med. 2007, 13:1324-1332.

33. Ross R: The pathogenesis of atherosclerosis: a perspective for 1990s. Nature 1993, 362:801-809.

34. Ying $\mathrm{HZ}$, Chen $\mathrm{Q}$, Zhang WY, Zhang $\mathrm{HH}$, Ma $\mathrm{Y}$, Zhang SZ, Fang J, Yu CH: PDGF signaling pathway in hepatic fibrosis pathogenesis and therapeutics (Review). Mol. Med. Rep. 2017, 16:7879-7889.

35. Aster RH: Pooling of platelets in the spleen: role in the pathogenesis of "hypersplenic" thrombocytopenia. J. Clin. Invest. 1966, 45:645-657.

36. Afdhal N, McHutchison J, Brown R, Jacobson I, Manns M, Poordad F, Weksler B, Esteban R: Thrombocytopenia associated with chronic liver disease. J. Hepatol. 2008, 48:1000-1007.

37. Lesurtel M, Graf R, Aleil B, Walther DG, Tian Y, Jochum W, Gachet C, Bader M, Clavien PA: Platelet-derived serotonin mediates liver regeneration. Science 2006, 312:104-107.

38. Alves R, Grimalt R: A Review of Platelet-Rich Plasma: History, Biology, Mechanism of Action, and Classification. Skin Appendage Disord. 2018, 4:18-24.

39. Nam GS, Nam KS: Arctigenin attenuates platelet activation and clot retraction by regulation of thromboxane A2 synthesis and CAMP pathway. Biomed. Pharmacother. 2020, 130:110535.

40. Barreto J, Karathanasis SK, Remaley A.; Sposito AC: Role of LOX1 as a Cardiovascular Risk Predictor: Mechanistic Insight and Potential Clinical Use. Arterioscler. Thromb. Vasc. Biol. 2021, 41:153-166. 